\title{
LOS DOGMAS SÉPTIMO Y OCTAVO DEL EMPIRISMO Y LA CONCEPCIÓN SEMÁNTICA DE LAS TEORÍAS CIENTÍFICAS
}

\section{THE SEVENTH AND EIGHTH DOGMAS OF EMPIRICISM AND SEMANTIC VIEW OF SCIENTIFIC THEORIES}

Miguel León Untiveros*

\begin{abstract}
RESUMEN
En el presente trabajo mostramos la existencia de una relación entre los dogmas del empirismo lógico con la concepción semántica de las teorías científicas. En especial, analizamos la relación entre los denominados séptimo y octavo dogmas del empirismo con la concepción semántica, y sostenemos que no es posible reformular el empirismo (liberal) a efectos de superar los dogmas en cuestión.
\end{abstract}

\section{PALABRAS CLAVE}

Dogmas del empirismo, concepción del enunciado, concepción semántica, método axiomático, Rudolf Carnap, Alfred Tarski, Patrick Suppes.

\begin{abstract}
In this paper we show there is a relationship between the dogmas of empiricism and the semantic view of empirical sciences. We analyze the special relationship between the so-called seventh and eighth dogmas of empiricism and semantic view of empirical sciences, and we claim there is no way to improve logical empiricism (in a liberal flavor) in order to overcome these dogmas.

\section{KEYWORDS}

Dogmas of empiricism, statement view, semantic view, axiomatic method, Rudolf Carnap, Alfred Tarski, Patrick Suppes.
\end{abstract}

Docente de la Universidad Católica Sedes Sapientiae: miguel.leon.u@gmail.com 


\section{Los dogmas del empirismo lógico.}

Desde que Willard van Orman Quine demostrara la existencia de dos dogmas en el empirismo lógico (1951), varios autores han seguido la crítica contra éste último, que en su momento era la concepción epistemológica dominante. Otro hito interesante, en el proceso de cambio de concepción en la epistemología, es el trabajo elaborado por Hilary Putnam quien en 1960, con su "What theories are not", donde además de darle al empirismo lógico el nombre de la concepción heredada (received view), ataca la distinción entre los términos observacionales y los términos teoréticos, y el concepto de interpretación parcial que se emplean en la concepción estándar. En este contexto de crítica, la tesis de la inconmensurabilidad de las teorías científicas propuestas por Paul Karl Feyerabend y Thomas Kuhn por separado, en 1958 y 1962 respectivamente, puso en real cuestionamiento al empirismo lógico. Sin embargo, ello aún no daba lugar a una nueva concepción de la ciencia, aunque ciertamente imponía nuevas condiciones a ser satisfechas.

Por otro lado, ya en los años de la segunda guerra mundial, el filósofo holandés Evert Willem Beth mostraba su insatisfacción con la creciente discrepancia entre la ciencia y la filosofía. Empleando el método semántico de Alfred Tarski, los desarrollos de este campo por Rudolf Carnap, y los trabajos sobre los fundamentos de la mecánica cuántica de Jon von Neumann (1932) y Martin Strauss (1938), Evert W. Beth propuso un análisis semántico de las mecánicas cuánticas y newtonianas (Suppe, 1989, p. 6). Asimismo, Patrick Suppes, quien en la década de 1950, junto con J. C.
C. McKinsey, A. C. Sugar y H. Rubin, publica una serie de trabajos en los que combinaba los métodos de la teoría de conjuntos de McKinsey y de Tarski.

En la década de los 1960s y 1970s se desarrollan en forma más consistente y con mayor velocidad los aspectos fundamentales de la concepción semántica, por autores como Frederick Suppe, Ronald N. Giere, Bas C. van Fraassen, Joseph D. Sneed (quien fue alumno de Suppes), Wolfgang Stegmüller, Wolfgang Balzer y Carlos Ulises Moulines, principalmente.

Este profundo cambio de la concepción filosófica de la ciencia se hizo con el fin de dar respuesta a las críticas formuladas contra la concepción estándar (i.e., empirismo lógico). Una forma de apreciar tales críticas, aunque no la única, es revisar la relación que existe entre los llamados dogmas del empirismo lógico y el surgimiento de la concepción semántica de las teorías científicas. En cuando refiere a los dogmas, estos ciertamente surgieron inicialmente en el seno del empirismo (Quine), pero no necesariamente llevaron a un cambio en la concepción (i.e., la concepción del enunciado). Fueron las formulaciones de otros dogmas los que acusan el cambio de concepción, esto es, hacia la concepción semántica. La lista de los dogmas del empirismo que hemos hallado son las siguientes.

(1) La distinción analítico/sintético.

(2) El reduccionismo del significado a la experiencia.

(3) El dualismo esquema/contenido.

(4) Las explicaciones científicas son argumentos.

(5) La idea de que las distinciones deben ser del tipo "o-todo-o-nada"1.

1 Este punto da lugar al principio de relevancia de las distinciones graduales el cual señala lo siguiente: "son filosóficamente relevantes las distinciones conceptuales que atienden sólo a diferencias de grado y no a diferencias absolutas en el objeto o dominio de estudio" (Moulines, 1982, pág. 32). 
(6) La sustitución de la ética por la metaética.

(7) La orientación a los métodos semántico y sintáctico, pasando por alto los conceptos pragmáticos.

(8) La concepción del enunciado de las teorías científicas.

(9) La inducción y el monismo metodológico.

(10) La distinción realismo/idealismo.

(11) El carácter nomológico de la causalidad.

(12) El dualismo hecho/valor.

(13) El negativismo empírico.

Nos guiamos por un criterio cronológico². Así, los dogmas (1) y (2) fueron formulados por Willard van Orman Quine en 1951 (en su trabajo "Two dogmas of empiricism"), el dogma (3) fue formulado por Donald Davidson en 1974 (en su trabajo "("On the Very Idea of a Conceptual Scheme"); el (4) es formulado por Wesley Salmon en su trabajo "A third dogma of empirismus" de 1975; en 1982 Carlos Ulises Moulines formula el (5); los formulados por Wolfgang Stegmüller en 1983 son del (6) - (10) (en su trabajo "Vom dritten bis sechsten (siebten?) Dogma des empirismus" ["Del tercer al sexto (¿o séptimo?) dogma del empirismo"|) $)^{3}$, el dogma ( 11 ) es formulado por John McDowell en 1985 (en su trabajo "Functionalism and Anomalous Monism") 4 . El dogma (12) es propuesto por Hilary Putnam en sus conferencias denominadas The Rosenthal Lectures (noviembre de 2000) ${ }^{5}$. Y, finalmente, el dogma (13) es propuesto por Saul Kripke en su trabajo (Yet another dogma of empiricism, 2014).

En la literatura pueden encontrarse otros dogmas que aun cuando no sean llamados de ese modo tienen el mismo carácter $^{6}$, y que deben ser tenidos como tales. La existencia de estos dogmas ha dado lugar a concebir al empirismo lógico como una doctrina obsoleta centrada en dogmas (Mormann, 2010, pp. 263-4) ${ }^{7}$.

De los dogmas antes indicados, los que nos interesan en esta ocasión son (7) y (8), que están vinculados directamente con el origen conceptual del enfoque semántico de las teorías científicas. Como se indicó, estos dogmas fueron formulados por Wolfgang Stegmüller (1983), y sostienen que la orientación del empirismo lógico considera que el concepto fundamental de explicación en la epistemología es únicamente semántico

2 Sin embargo, existe de hecho un criterio, por decirlo así, "argumentativo", en el sentido de que aceptar, por ejemplo, los dogmas (1) y (2) presupone aceptar el dogma (3) (Davidson, 1973-1974, p. 11). En este trabajo, no hemos seguido tal criterio pues no todos los dogmas están relacionados de ese modo.

3 En este trabajo, Wolfgang Stegmüller ignora el tercer dogma formulado por Donald Davidson en 1974. Cabe indicar que el dogma (6) fue también enunciado por Stegmüller en otro trabajo del mismo año (1983, pp. 2-10).

4 En este trabajo, John McDowell ignora los dogmas formulados por Wolfgang Stegmüller en 1983.

5 Una versión revisada se encuentra en (Putnam, 2002).

6 Por ejemplo, Jesús Antonio Coll Mármol critica la distinción entre pensamiento activo y pensamiento pasivo (i.e., la idea de que debe haber un elemento epistemológico que pasivamente lleva al mundo a nuestra mente, permitiendo que nuestros pensamientos esté en fricción con el mundo) (Coll Mármol, 2007, pp. 46-9).

7 No obstante, para las concepciones semánticas de las teorías científicas permanece como tarea la reconstrucción racional de la ciencia, como lo propuso en su momento el empirismo lógico (i.e., (Carnap, 2005 (1928)), (Reichenbach, 1938), entre otros). La diferencia consiste tanto en la concepción cuanto en el método. Por lo primero, para la concepción semántica la reconstrucción de las teorías no se centra en los enunciados de ésta sino en los predicados (modelos). Por lo segundo, la concepción semántica no emplea exclusivamente una lógica de primer orden sino además lógicas de nivel superior y teorías de conjuntos (da Costa E French, 2003, p. 25). 
y metodológicamente sintáctico, donde los términos pragmáticos son ignorados (Stegmüller, 1983, pp. 234-5).

\section{El método axiomático.}

Antes de seguir con nuestro tema, es conveniente aclarar que si bien ha habido un declive en la larga búsqueda de bases sólidas del conocimiento, esto no quiere decir que se haya dado fin a la importancia de los métodos formales en la filosofía de la ciencia (Suppes, 1993 [1979], p. 5). Y esto importa para el caso del método axiomático. Uno de los logros más importantes de fines del siglo XIX es el refinamiento del método axiomático. Como es sabido, este método fue desarrollado por Euclides (siglos IV - III a. C.) en sus Elementos, pero concebía a los conceptos primitivos como posibles de ser definidos ${ }^{8}$ así como los postulados se consideraron verdades evidentes. Estas características de la axiomática euclídea son superadas por la axiomática de David Hilbert, quien en su obra los Fundamentos de la Geometría (1899), los conceptos primitivos no son definidos sino como puntos de partida y los axiomas (o postulados o principios) son propiedades sin definición ni demostración que se formulan en términos de los conceptos primitivos. Hilbert adoptó una nueva concepción de axioma y concepto primitivo formulados por Moritz Pasch ${ }^{9}$, quien a diferencia de los clásicos griegos concibió la separación entre los axiomas y los conceptos primitivos con el sentido (o significado) de los mismos, considerando que esto último tiene un marcado segundo lugar, y hasta accesorio. A ello, David
Hilbert exigía que el sistema de axiomas cumpla con las siguientes propiedades (Reid, 1970, p. 63):

- Compleción: el sistema axiomático debe ser suficiente para probar todos los enunciados del mismo.

- Independencia: Que cada axioma sirva para probar por lo menos un teorema.

- Consistencia (simple o de negación): que no se incurra en contradicción, esto es que no se deriva de los axiomas la afirmación y negación de un enunciado.

Adicionalmente, en la actualidad se exige que el sistema axiomático cumpla a estas propiedades (Cassini, 2006, págs. 95-111):

- Decidibilidad: Que exista en el sistema un procedimiento mecánico de decisión para establecer si una fórmula pertenece o no a ese sistema.

- Satisfacibilidad: Un sistema axiomático $S$ es satisfacible, si por lo menos tiene un modelo.

- Categoricidad: Un sistema axiomático $S$ es categórico si y sólo si $S$ es consistente y todos sus modelos son isomorfos entre sí $^{10}$.

A su vez para Hilbert, el método axiomático tenía como fin la unificación, el ordenamiento, la claridad y simplicidad del conocimiento (Reid, 1970, pp. 63-67, 85). Cabe indicar que esta posición de Hilbert constituye una reacción contra la doctrina matemática que sostenía

8 Por ejemplo, en el libro I de los Elementos, Euclides define el punto como lo que no es parte y la línea como lo que es largo sin anchura (Euclid, 1908, p. 154).

9 La concepción de Moritz Pasch fue expuesta en su obra Vorlesungen über neuere Geometrie (Lecciones sobre geometría moderna], de 1882. Sobre este punto ver (Freudenthal, 1962) y (Torretti, 1993).

10 Para una referencia filosófica del concepto de categoricidad, puede verse: (Corcoran, 1980), (Awodey E Reck, 2002), (Awodey \& Reck, 2002), entre otros. 
Ignoramus et ignorabimus - somos ignorantes y permanecemos ignorantes - sostenida por el científico (médico y fisiólogo) alemán Emil duBois-Reymond (1818-1896), y que Hilbert sintetizó así: Wir müssen wissen. Wir werden wissen - iDebemos saber y lo sabremos! (Hilbert, 1996 [1930], p. 1165).

Así, la profundización en los fundamentos del conocimiento nos lleva a identificar que la base de la estructura conceptual existen algunas proposiciones que resultan suficientes y que en coordinación con los principios lógicos puede construirse la estructura conceptual íntegra. A tales proposiciones se les llama axiomas del campo de conocimiento en concreto, y que el progresivo desarrollo del mismo consiste en una mayor construcción lógica de los conceptos antes mencionados (Hilbert, 1996 [1918], p. 1108).

Sin embargo, el método axiomático antes descrito en el siglo XX ha sido objeto de restricciones en cuanto a su alcance, límites y rigurosidad. Sobre los alcances del método axiomático, se indica que el mismo sólo debe utilizarse en la ciencia cuando ésta haya conseguido una suficiente cantidad de descubrimientos científicos y no antes, pues ello tendría el efecto de desviar o restringir la investigación empírica. Y en cuando a los límites se ha señalado lo siguiente: (1) la existencia de elementos de arbitrariedad, esto es que se ha hallado que existen caracterizaciones equivalentes del mismo objeto matemático, así se tiene el caso de la teoría de retículos que puede formalizarse tanto mediante el concepto de igualdad como un predicado fundamental; (2) la existencia de deficiencias del método axiomático, que son de dos tipos (a) del tipo sintáctico, constituido por la imposibilidad de probar formalmente cierto teoremas que son verdaderos de cara a la consistencia del sistema axiomático formal, como lo ha señalado Gödel, y (b) del tipo semántico, que hace referencia a la existencia de modelos no estándares, i.e., que los modelos difieren de la estructura a ser descrita por un sistema de axiomas, como lo indicara primero Skolem (Bernays, 1967).

No obstante la ocurrencia de estos embates contra el método axiomático, puede decirse que el proceder axiomáticamente no es más que pensar con conciencia sin incurrir en la ingenuidad de creer en los dogmas (Hilbert, 1996 [1922], p. 1120). Y por ello es una forma de expresar el razonamiento de un modo más ordenado, claro y simple que con respecto a otros tipos de pensamiento. Y en ese sentido se mantiene vigente el propósito fundamental de Hilbert. Las dos corrientes epistemológicas más importantes del siglo XX y en lo que va del presente (i.e. el empirismo lógico y la concepción semántica de las ciencias) han adoptado como método de sus filosofía el método axiomático. Y si bien este aspecto los hace, en un sentido, semejantes, inmediatamente debemos indicar que el modo en que emplean dicho método a la vez los hace diferentes. Este punto lo explicaremos más adelante, por ahora baste decir que el empirismo lógico empleó el método semántico en un lenguaje de primer orden pues concibe a la ciencia como un conjunto de enunciados (sin dar un lugar formal a los aspectos pragmáticos del desarrollo de la ciencia), mientras que la concepción semántica concibe a la ciencia como enunciados predicativos expresados en teoría de conjuntos (en cuya formalización se incluyen los aspectos pragmáticos del desarrollo científico) ${ }^{11}$.

11 Para una visión histórica y actual del método axiomático véase (Rodin, 2014), en especial los capítulos 2 - 5. 


\section{La concepción del enunciado de las teorías científicas.}

A esta concepción Hilary Putnam la denominó la concepción heredada (received view), en su trabajo denominado "What theories are not" (Putnam, 1962), donde acusa a la posición del Círculo Viena de haber heredado en forma acrítica la distinción entre enunciados observacionales y enunciados teóricos, así como el concepto de interpretación parcial $^{12}$. Esto es que la denominación de "concepción heredada" o "concepción recibida" 13 hace referencia a una actitud acrítica de ciertos puntos de partida heredados o recibidos por empirismo lógico. Asimismo, se la ha denominado la concepción del enunciado (statement view), por Wolfgang Stegmüller, enfatizando su enfoque en los términos en los que se formulan las leyes científicas.

En medio de esta multiplicidad de términos ${ }^{14}$, es necesario tener una definición clara de lo que entendemos por la concepción enunciativa de la ciencia. Siguiendo a Frederick Suppe (1977, pp. 1617), las características de esta concepción (sintáctica o del enunciado) son:

La reconstrucción de las teorías se realiza en forma axiomática en un lenguaje matemático $L$, que tiene las siguientes condiciones: i. La teoría es formulada en una lógica matemática de primer orden con igualdad, $L$.

ii. Los términos no lógicos o constantes de $L$ son divididos en tres clases diferentes, llamados vocabularios:
a. El vocabulario lógico, que consiste en las constantes lógicas (incluyendo términos matemáticos).
b. El vocabulario observacional, $V_{O}$, que contiene términos observacionales.
c. El vocabulario teorético, $V_{T}$, que contiene los términos teóricos.

iii. Los términos en $V_{O}$ son interpretados en referencia a objetos físicos directamente observables o atributos observables directamente de objetos físicos.

iv. Existe un conjunto de postulados teóricos $T$ cuyos únicos términos no lógicos pertenecen a $V_{T}$.

v. Los términos en $V_{T}$ se dan por definición explícita, en términos de $V_{O}$ mediante las reglas de correspondencia $C$ - esto es, que para cada término ' $F$ ' en $V_{T}$, debe darse una definición para ésta de la siguiente forma:

$$
\forall x(F x \equiv O x)
$$

12 No obstante, existen otras denominaciones con las que califica a esta posición epistemológica, a saber: concepción sintáctica (syntactic approach), término empleado por ejemplo por Bas C. van Fraassen.

13 Esta sería la traducción literal de "received view", la cual es empleado en nuestro medio por Luis Piscoya Hermoza, así en sus clases de epistemología en la Universidad Nacional Mayor de San Marcos (2013 - I).

14 En la literatura, hemos registrado hasta ocho términos empleados para referir lo mismo: (i) positivismo lógico, (ii) empirismo lógico, (iii) concepción tradicional (e.g., (Balzer, Moulines, E Sneed, 1987)), (iv) concepción estándar (e.g. (da Costa E French, 2003), (Hands, 2001)) (v) concepción heredada (e.g., (Díez E Moulines, 2008)), (vi) concepción recibida, (vii) enfoque sintáctico (e.g., (van Fraassen, 1980)), (viii) concepción de enunciado (e.g., (Stegmüller, 1976 (1973))), (IX) neopositivismo lógico (Kraft, 1966 [1950]), y (x) concepción ortodoxa (Feigl, 1970), (Hughes, 2010). 
Donde ' $O x^{\prime}$ ' es una expresión de $L$ conteniendo símbolos únicamente pertenecientes a $V_{O}$ y, posiblemente, otros del vocabulario lógico.

No obstante, esta caracterización muy conocida de la concepción heredada, no hace énfasis en que ella también tiene un aspecto semántico ${ }^{15}$. Así, si bien puede diferenciarse dos periodos en la obra de Carnap, uno sintáctico (hasta 1934) y otro semántico (desde 1935), Pablo Lorenzano, siguiendo a Jaakko Hintikka, señala que no es sostenible una distinción estricta entre ambos periodos, pues en la obra más significativa del periodo sintáctico, Logische Syntax der Sprache (1934) puede considerarse en gran parte semántico (Lorenzano, 2012, pág. 110). Es el mismo Frederick Suppe que subsana tal omisión (Suppe, 1989, pp. 39-41). Veamos:

Las teorías científicas son tales que pueden tener una formulación canónica que satisfaga las siguientes condiciones:

(1) Existe un lenguaje de primer orden $L$ en términos del cual la teoría es formulada, y un cálculo $K$ definida en términos de $L$.

(2) Las constantes primitivas descriptivas o no lógicas (i.e., los "términos") de $L$ se bifurcan en dos clases distintas:

(a) $V_{O}$ que contiene sólo términos de observación, y

(b) $V_{T}$ que contiene términos teoréticos o que no de observación.

$V_{O}$ debe contener al menos una constante individual.
(3) El lenguaje $L$ está divido en sub lenguajes y el cálculo $K$ está divido en sub cálculos, en la siguiente manera:

(a) El lenguaje de observación, $L_{O}$, es un sub lenguaje de $L$ que no contiene cuantificadores o modalidades y contiene los términos de $V_{O}$ pero ninguno de $K_{O}$. El cálculo asociado $K_{O}$ es una restricción de $K$ para $L_{O}$ y debe ser tal que cualquier término-no $V_{O}$ (i.e., los términos no-primitivos) en $L_{O}$ son explícitamente definidos en $K_{O}$. Adicionalmente, $K_{O}$ debe admitir al menos un modelo finito.

(b) El lenguaje de observación lógicamente extendido, $L_{O}^{\prime}$ no contiene términos-. $V_{T}$ Podría considerarse que está formado desde $L_{O}$ agregándosele los cuantificadores, las modalidades, etc., de $L$ para $L_{O}$. Su cálculo asociado, $K_{O}^{\prime}$, es la restricción de $K$ para $L_{O}^{\prime}$.

(c) El lenguaje teorético, $L_{T}$, es aquél sub lenguaje de $L$ que no contiene términos- $V_{O}$; su cálculo asociado $K_{T}$ es la restricción de $K$ para $L_{T}$.

Estos sub lenguajes juntos no son exhaustivos con respecto a $L$, pues $L$ también contiene enunciados mixtos, i.e., aquellos en los cuales concurren al menos un término- $V_{T}$ y término $-V_{O}$. Asimismo, se asume que cada uno de los sub lenguajes

15 Empleamos este término en sentido lato, sin embargo, resulta útil saber que tiene, por lo menos, dos significados: uno en el que semántica hace referencia al estudio de las propiedades de los enunciados que no varían en razón de su uso, en un segundo sentido hace referencia al estudio de las propiedades de éstos en relación con el contexto de su uso, a éste último sentido también se conoce como pragmática. 
antes indicados tiene su propio inventario de predicados o variables funcionales y $L_{O}$ y $L_{O}^{\prime}$ tienen el mismo inventario, el cual es distinto al de $L_{T}$.

(4) A $L_{O}$ y sus cálculos asociados se les da una interpretación semántica que cumple con las siguientes condiciones:

(a) El dominio de observación consiste en las entidades observables concretas tales como eventos, cosas o cosamomentos observables; las relaciones y las propiedades de la interpretación deben ser directamente observables.

(b) Cada valor de cualquier variable en $L_{O}$ debe ser designado mediante una expresión en $L_{O}$.

Se sigue que cualquiera de las interpretaciones de $L_{O}$ y $K_{O}$, a las que se les aumente apropiadamente las reglas de verdad, constituirán una interpretación de $L_{O}^{\prime}$ y $K_{O}^{\prime}$. Podemos construir interpretaciones de $L_{O}$ y $K_{O}$ como si fuesen interpretaciones semánticas parciales de $L$ y $K$, y requeriremos que $L$ y $K$ no tengan otra interpretación semántica empírica que la provista por tales interpretaciones semánticas.

(5) La interpretación parcial de los términos teoréticos y de los enunciados de $L$ conteniéndolo es provista por los dos tipos de enunciados siguientes: los postulados teoréticos $T$ (i.e., los axiomas de la teoría), en los cuales sólo ocurren los términos de $V_{T}$, y las reglas de correspondencia o postulados $C$, que son enunciados mixtos. Las reglas de correspondencia
$C$ deben satisfacer las siguientes condiciones:

(a) El conjunto de reglas $C$ debe ser finito.

(b) El conjunto de reglas $C$ debe ser lógicamente compatible con $T$.

(c) $C$ no contiene términos extra lógicos que no pertenezcan a $V_{O} \circ V_{T}$.

(d) Cada regla en $C$ debe contener por lo menos un término $V_{O}$ y por lo menos un término $V_{T}$ esencialmente o no debe ser vacío.

Sea $T$ la conjunción de los postulados teoréticos y $C$ la conjunción de las reglas de correspondencia. Entonces, la teoría científica basada en $L, T$ y $C$ está constituida por la conjunción de $T$ y $C$ y se designa como ' $T C$.

Nótese que la condición (4) permite la posibilidad de sistemas semánticos alternativos (o interpretaciones) para $L_{O}$ los cuales pueden diferir de las designata por los términos $-V_{O}$. Sin embargo, la concepción heredada pretende entender que existe un conjunto fijo de designata para los términos de $V_{O}$, y así las restricciones deben ser impuestas sobre la clase de las interpretaciones admisibles. Si asumimos que se haya especificado un conjunto fijo de reglas de designación para los términos$V_{O}$, entonces diremos que la clase de los sistemas semánticos que usan estas reglas son sistemas semánticos permisibles para $L_{O}$, y la clase de interpretaciones que ellos especifican son las interpretaciones permisibles para $L_{O y} K_{O}$. Nótese que es posible que se den diferentes interpretaciones permisibles, dado que las reglas de designación para las variables predicativas pueden diferir. Las clases de sistemas semánticos permisibles e interpretaciones para $L_{O}^{\prime}$ y $K_{O}^{\prime}$ son definidas análogamente. 
Una forma de representar lo anterior, de modo más simple fue ofrecida por Herbert Feigl (1970, p. 6) con el siguiente gráfico:

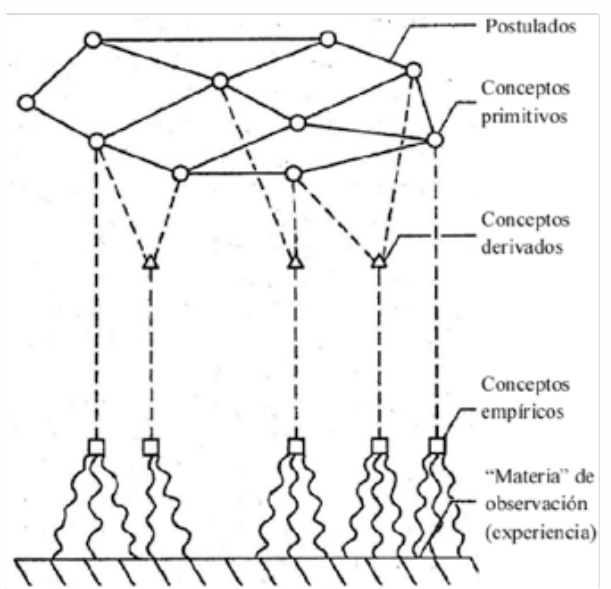

Donde:

O: son los conceptos primitivos.

$\Delta$ : son los conceptos derivados.

$\square$ : son los conceptos empíricos.

Como indica el gráfico, los conceptos teoréticos básicos (primitivos) son definidos implícitamente por los postulados. Los conceptos primitivos, o más usualmente los conceptos derivados definidos explícitamente en términos de los primitivos, están vinculados por la reglas de correspondencia en referencia a los puntos de la observación, e.g., en las ciencias físicas usualmente son las cantidades medibles como la masa, la temperatura y la intensidad de la luz. Estos conceptos empíricos, a su turno, son operacionalmente definidos, i.e., mediante la especificación de las reglas de observación, medición, experimentación o diseño estadístico que determinan y delimitan su aplicabilidad y aplicación.
Como hemos visto, en el empirismo lógico se da un nivel semántico, como se indican en los puntos (4) y (5) de la formulación hecha por Frederick Suppe (1989). Por ello no resulta exacto decir que el empirismo lógico sea ajeno a la semántica ${ }^{16}$. No obstante, este enfoque, como se puede apreciar tiene como centro de análisis los enunciados de las leyes científicas, prescindiendo de todo aspecto pragmático (e.g., la inconmensurabilidad). De forma que, desde esta concepción, tendríamos que admitir que cuando haya un cambio de sintaxis en la axiomatización de una teoría ello daría lugar a un "progreso" o (por lo menos) "cambio" en la ciencia. Lo cual no se condice con la práctica científica. En esto consiste el séptimo dogma del empirismo. De igual manera, desde esta concepción, los aspectos pragmáticos, como la inconmensurabilidad, no tendrían lugar en el análisis de las teorías científicas. Esto, tampoco, se condice con la práctica científica. A esta última actitud se le denomina el séptimo dogma del empirismo. Como se señaló anteriormente, ambos dogmas fueron expresados por Wolfgang Stegmüller en $1983^{17}$. Hasta aquí nuestra caracterización del empirismo lógico.

\section{La concepción semántica de las teorías científicas.}

El principal cambio en la concepción de la ciencia, operado en la segunda mitad del siglo veinte, está dado por la concepción semántica. Esta no es sino un conjunto (una familia) de concepciones epistemológicas. Según D. Wade Hands (2001, p. 311 ), las versiones básicas de la concepción semántica son:

16 Para hacer tal cosa, debe hacer una mayor precisión del término semántica en relación con el uso de los enunciados, como indicamos en la nota anterior. Este tema no abordamos en el presente trabajo.

17 El hecho que en este trabajo tales dogmas sean el séptimo y octavo, se debe a la ordenación cronológica que hemos señalado, anteriormente. 
1. La de teoría de modelos de Ronald N. Giere.

2. La de la fase espacial de Frederick Suppe.

3. La del estado espacial de Elisabeth Lloyd y Bas C. van Fraassen.

4. El estructuralismo metateórico (Joseph D. Sneed, Wolfgang Stegmüller, Wolfgang Balzer, Carlos Ulises Moulines).

Siguiendo un ejemplo de D. Wade Hands dado para visualizar mejor la diferencia entre la concepción estándar (statement view) y la concepción semántica de la ciencia tenemos: según la concepción estándar, las teorías científicas son simplemente conjuntos de enunciados; los cuales pueden ser verdaderos o falsos, corroborados o falseados sobre la base de otros enunciados acerca de los datos empíricos; no obstante en todo caso se trata siempre de enunciados. Por otro lado, de acuerdo con la versión semántica, una teoría científica únicamente define un predicado. Por ejemplo, la mecánica de Newton define el predicado "es un sistema newtoniano", mientras que la economía keynesiana define el predicado "es una economía keynesiana". Tales predicados no pueden ser verdaderos o falsos ni proporcionan predicciones. La manera en que las afirmaciones científicas son generadas a partir de las teorías científicas es formular una hipótesis empírica de la forma "x es un ejemplo de la teoría científica T". Así, el enunciado "nuestro sistema solar es un sistema newtoniano" es una hipótesis científica asociada a la mecánica de newton, mientras que el enunciado "la economía de los Estados Unidos de América de 1933 fue una economía keynesiana" es una hipótesis empírica asociada con la economía keynesiana. Las hipótesis científicas a diferencia de las teorías científicas pueden ser verdaderas o falsas.
Los predicados antes indicados son llamados modelos. Conviene aclarar este punto. Siguiendo a Wolfgang Balzer, Carlos Ulises Moulines y Joseph Sneed (1987, pp. 2-3), las unidades básicas del análisis semántico de las teorías científicas son los modelos y no los enunciados, como lo es para el enfoque sintáctico de los fundamentos de la ciencia. En la concepción semántica, el término "modelo" tiene el sentido de la lógica moderna y la teoría de modelos. Los autores mencionados señalan que en el lenguaje ordinario y en contextos informales dentro de las ciencias empíricas, el término "modelo" es usado con referencia a la relación que hay entre el "dibujo" de algo y la cosa "representada". Así, el término "modelo" es algunas veces empleado en el sentido de dibujo y otras veces en el sentido de la cosa representada. Añaden, que los científicos empíricos tienden a usar "modelo" en el sentido de "dibujo", como cuando dicen que cierto conjunto de ecuaciones "es el modelo" de algún fenómeno sub atómico o de ciertas situaciones de los mercados reales. Los lógicos y los matemáticos consistentemente usan "modelo" en el sentido de la cosa representada por un dibujo (que vendría ser la teoría). Siendo el caso que el segundo significado del término "modelo" está bien establecido y claramente definido en las ciencias formales, es el que adopta la concepción semántica. De este modo, tenemos que a pesar de que se diga que ciertas ecuaciones son el modelo de un fenómeno sub atómico o económico, para la concepción semántica el fenómeno sub atómico o económico son los modelos de la teoría representada por esas ecuaciones.

Un ejemplo usualmente empleado para graficar el uso semántico del término "modelo" en el sentido de "la cosa representada" es el siguiente: una mujer 
que es el modelo de una pintura. En este caso, la modelo es la persona representada y la pintura es el dibujo de ella. El uso de los lógicos, y el semántico del término "modelo", es consistente con el uso artístico. Entonces, para la concepción semántica el término "modelo" es empleado en el sentido de la ocurrencia de un hecho ejemplar a seguir $\left(2002\right.$, p. 17) ${ }^{18}$. El modelo es una realidad socialmente constituida, cuya realidad no va más allá de lo concebido por la comunidad científica (Giere, 1988, p. 78). Siguiendo a Suppes, "el modelo es una identidad no-lingüística en la que una teoría es satisfecha" (1960, pp. 3-4). Esta concepción semántica de modelo se basa esencialmente en el trabajo de Alfred Tarski, quien en su definición de modelo indica: "Se llama modelo de $\mathrm{T}$, a la realización posible en la que todas las teorías válidas de una teoría T son satisfechas" (Tarski, 1971 (1953), p. 11). Éste es el fundamento del cambio operado en la epistemología y que dio lugar a la concepción semántica.

Por otro lado, las críticas contra el empirismo lógico parecieran haber desacreditado a la axiomatización de las teorías científicas. De acuerdo con la concepción heredada de teoría científica, "una teoría es un cálculo axiomático dada una interpretación observacional parcial mediante un conjunto de reglas de correspondencia, así la teoría es vista como una entidad lógicolingüística." (da Costa E French, 2003, p. 23). Esto habría sido la causa del descrédito de la axiomatización de las teorías. Sin embargo, Newton da Costa y Steven French, siguiendo a Suppes ${ }^{19}$, sostienen que la axiomatización es "un componente fundamentalmente importante en la filosofía de la ciencia, debido a su rol en la introducción de claridad con respecto a los conceptos básicos de la teoría, su ayuda para la comparación de las teorías (...), por la forma como se puede abrir la teoría a técnicas matemáticas potencialmente fructíferas $y$, sí, por su utilidad en resolver ciertas disputas filosóficas" (da Costa E French, 2003, p. 23).

No obstante ello, la axiomatización no procede meramente de modo lingüístico o sintáctico. Pues si fuera ese el caso, como ocurrió en la concepción heredada, cualquier cambio en las reglas de correspondencia (métodos experimentales o procedimientos de medición) darían lugar a una nueva teoría (i.e., séptimo dogma del empirismo). Y tal situación no tiene su contrapartida en la práctica científica (Suppe, 1989, pp. 4-5). Si bien resulta vano decir que la teoría de la relatividad general es la misma sea que se la exprese en inglés o portugués, no es vano decir que una teoría sigue siendo la misma cualquiera sea la forma lógicolingüística de su axiomatización (da Costa E French, 2003, p. 24).

Siguiendo a C. Ulises Moulines y José A. Díez (1994, p. 276), los puntos en los que convergen las diferentes concepciones dentro de la familia semántica son los siguientes:

a) Una teoría empírica no es una entidad lingüística, concretamente, no es un conjunto de enunciados axiomáticos o una conjunción de estos. Sino que es un tipo de entidad matemática.

b) El componente más básico de la entidad de una teoría una clase

18 La primera edición de este libro de Suppes se titula: Set-theoretical Structures in Science (1962) y sólo circuló en forma limitada en ejemplares mimeografiados.

19 Patrick Supes señala que "dada una teoría axiomatizada de la medición de una cantidad empírica como la masa, distancia o la fuerza, la labor matemática es probar el teorema de representación de los modelos de la teoría que establece, en términos generales, que cualquier es isomórfico a algunos modelos numéricos de la teoría" (Suppes, 1967, pp. 58-9). 
de estructura, específicamente, la identidad de la teoría es la clase de modelos en el sentido de Tarski.

c) Típicamente, esta clase de modelos no es un simple, ni siquiera una clase de estructuras isomórficas. La categoricidad ${ }^{20}$ no es algo que pueda encontrarse en las teorías empíricas desarrolladas y ni siquiera es un desiderátum. El desiderátum para las teorías empíricas es más bien es tener una clase heterogénea genuina de aplicaciones empíricas.

d) La manera más conveniente de elegir los modelos esencialmente caracterizadores de la identidad de una teoría es a través de los predicados de teoría de conjuntos. i.e., definiendo predicados de segundo orden en una teoría intuitiva [naïve] de conjuntos.

La concepción semántica, en su versión estructuralista, tiene asunciones ontológicas expresamente reconocidas, las que son (Moulines, 1996, p. 3):

- Las teorías científicas existen, por lo menos en tres sentidos: teoría-elemento, teoría-red y teoría-holón.

- Las teorías científicas son objetos culturales de una clase más abstracta, en el sentido de que no están localizadas espaciotemporalmente en la forma como lo están los objetos físicos. Tienen un estatus ontológico similar al del lenguaje, las sinfonías, los programas de computadora, entre otros.

- Al modo de otros objetos culturales más abstractos, las teorías científicas tienen una estructura profunda. Es decir, que su criterio de identidad y sus componentes esenciales no pueden ser detectados en forma directa a través de la observación de su apariencia superficial de los objetos que la ejemplifican (e.g., textos científicos). Explicitar dicha estructura no es una tarea trivial.

- Las teorías son entidades genidénticas, esto es que las teorías nacen, se desarrollan y se extinguen.

- Las teorías científicas no son mónadas, sino están esencialmente relacionadas con objetos fuera de ellas. Al menos una parte del mundo externo consiste en otras teorías. Esto quiere decir que existen relaciones inter teóricas y que pertenecen a la esencia de las teorías científicas.

De este modo, la concepción semántica de la ciencia ya no concibe la explicación en la epistemología en

20 Una Teoría $\mathbb{T}$ se llama categórica (con relación a una semántica dada) si para todos los modelos M, N de $\mathbb{T}$ hay isomorfismo entre M y N (Awodey $E$ Reck, 2002, p. 3). Los autores antes indicados señalan que informalmente, la idea es que $\mathbb{T}$ tiene 'esencialmente un solo modelo'. Son ejemplos familiares, la aritmética de Peano, con el usual axioma de inducción de segundo orden, y la teoría de segundo orden de los campos ordenados completos. En contraste de que la versión de primer orden de las mismas en no son categóricas.

Históricamente, el término "categórico" fue explicitado por Oswald Veblen y Edward V. Huntington, el primero lo empleó en su trabajo (A system of axioms for Geometry, 1904), donde en una nota a la página 346, señala que éste término le fue sugerido por John Dewey. Edward V. Huntington explica este concepto en su trabajo (A Complete Set of Postulates for the Theory of Absolute Continuous Magnitude, 1902). Oswald Veblen, en (1905, p. 168), en una nota a pie de página, da una definición equivalente a la indicada. Este concepto es tomado por Alfred Tarski (1956 (1931), p. 174). 
un sentido únicamente lingüístico y metodológicamente sintáctico, donde los términos pragmáticos son ignorados. Ello debido a que tal situación fue el centro de la crítica de la tesis de la inconmensurabilidad de las teorías científicas de Paul Karl Feyerabend y Thomas Kuhn. Sino que por el contrario, para la concepción semántica las teorías se conciben como entidades que evolucionan y hasta son sustituidas. Así, para la concepción semántica (en su versión estructuralista), puede haber por lo menos cuatro tipos fundamentales distintos de desarrollo de las teorías científicas (Moulines, 2011 ) $^{21}$ :

a) Emergencia o cristalización de teorías. Trata de la fase inicial de una disciplina. Por ejemplo, la teoría de conjuntos de Georg Cantor surgido entre 1870 e inicios de la década de 1880s.

b) Evolución de teorías. Trata de cuando se añaden o suprimen algunos elementos teóricos sin perder su identidad esencial, el cual está representado por un núcleo teórico básico. Por ejemplo, la astronomía ptolemaica desde el s. II d.C. hasta mediados del siglo XVI.

c) Incorporación o incrustación de una teoría en otra. En este caso los modelos de una teoría se incorporan o incrustan (aproximadamente y quizás no completamente) en los modelos de una nueva teoría, más compleja. Por ejemplo, la incorporación de la mecánica cartesiana del choque en la mecánica newtoniana. d) Suplantación de una teoría por otra acompañada de inconmensurabilidad parcial. Una teoría aplicable a un dominio de cosas es sustituida por otra que explica más o menos el mismo dominio de cosas. Por ejemplo, la suplantación de la teoría del flogisto por la teoría del oxígeno de Lavoisier.

Siguiendo a Wolfgang Stegmüller, decimos que la necesidad de precisión y claridad que nos brindan los aspectos lógicos, está dada por el hecho histórico consistente "en el increíble número de interpretaciones equívocas y rivales del libro de Kuhn [1962], desde las primeras revisiones hasta los recientes 'debates sobre paradigmas'. [Y que] Podrian haberse evitado gran parte de esos debates con una utilización adecuada de la lógica y de la teoría de conjuntos" (Stegmüller, 1984 (1978), pág. 262)²2.

A efectos de establecer mejor las diferencias entre la concepción epistemológica empirista y la concepción semántica (en su versión estructuralista), a la primera se la ha llamado "la concepción del enunciado" (statement view), en adelante "st. $\boldsymbol{v}$ ". De acuerdo con Wolfgang Stegmüller (1979), este término tiene cuatro significados diferentes:

(i) En un primer sentido, $s t . v_{1}$ hace referencia al enfoque formal del lenguaje, y se llama también enfoque de Carnap. Mientras que el enfoque semántico (de corte estructuralista), en adelante "non - st. $\boldsymbol{v}^{\text {'", }}$, refiere a un enfoque que hace un uso informal de la teoría de conjuntos y la lógica,

21 En el artículo citado así como en (Balzer, Moulines, E Sneed, 1987) pueden hallarse formulaciones rigurosas de los tipos de desarrollo de las teorías científicas.

22 Recordemos que Margaret Masterman detectó 21 definiciones del término paradigma en el libro The Structure of Scientific Revolutions de Thomas S. Kuhn (Masterman, 1970, pp. 61-5). 
al que también se le denomina el enfoque de Suppes.

(ii) En un segundo sentido, $s t . v_{2}$ requiere que los aspectos empíricos de las teorías sean representados a través de clases infinitas de enunciados. Mientras que para el $n o n-s t . v_{2}$ tal aspecto debe ser representado mediante el enunciado corregido de Ramsey. Si bien el primer significado st. $v_{1}$ es lógicamente posible, no sucede lo mismo con $s t . v_{2}$.

(iii) En un tercer sentido, non $-s t . v_{2,5}$ el tiene que ver con la forma en las leyes fundamentales o especiales entran o llegan a ser parte de los aspectos empíricos.

(iv) En un cuarto sentido, el $s t . v_{3}$ tiene un carácter rígido y apartado de las aspectos pragmáticos de la ciencia, siendo que sucede lo contrario con el non $-s t$. $v_{3}$, el cual incluye en su análisis tales aspectos pragmáticos.

Sin embargo, existe una interesante discusión en la interpretación del programa carnapiano. Andoni Ibarra distingue entre un Carnap el cual postula la teoría del doble nivel del lenguaje, por el que se entiende que un término teórico $\mathrm{t}$ es significativo si existe un supuesto $A$ que comprenda t tal que a partir de $A$ y de supuestos adicionales que comprendan otros términos teóricos considerados significativos se puede derivar, con la ayuda de los postulados y las reglas de correspondencia, un enunciado observacional que no puede obtenerse sin el supuesto $A$ (Ibarra, 2012, pág. 96) ${ }^{23}$. Según Ibarra, por otro lado, puede identificarse un Carnap 2 que plantea el problema de la estructura de las teorías como el problema de ofrecer una elucidación razonable de la relación de la parte teórica, formal o matemática, de una teoría con la parte que expresa el mundo. Así, en Carnap "subyace una caracterización de las teorías empíricas que ofrece para la significatividad de los términos teóricos un enfoque holista, pragmatista y relativo a la teoría" (Ibarra, 2012, pág. 98)24. No obstante, Ibarra reconoce que la gran diferencia de la concepción semántica con respecto a la del statement of view (en su versión carnapiana ( 1 y 2)) es que se ha asumido "también como tarea de la filosofía el estudio de las cuestiones externas prácticas - pragmáticas que, sin rechazarlas como las metafísicas, no se consideraban genuinas de la agenda de los problemas filosóficos. Esto es algo que ni siquiera el Carnap más liberal llegó nunca a proyectar" (Ibarra, 2012, pág. 100).

\section{La no trivialización del concepto "dogma del empirismo". ${ }^{25}$}

Finalmente, no es nuestra intención defender la trivialización del concepto "dogma del empirismo". Y a tal efecto, no creemos que el hecho de que los dogmas del empirismo sean por lo menos once, de ello se siga que se haya trivializado el concepto de "dogma del empirismo". Veamos. Supongamos el caso del concepto hombre, éste no es en absoluto trivial por el hecho de que,

23 Esta es la concepción de Carnap en (Carnap, 1956).

24 Este es el Carnap en (Beobachtungssprache und theoretische sprache, 1958) y (Autobiografía intelectual, 1992 (1963)). Para una discusión sobre la obra de Rudolf Carnap ver (Ibarra, La interpretación estructuralista del Statement View, 2012), (Lorenzano, 2012) y la réplica de Ibarra (2012).

25 La inclusión de esta parte del trabajo, surgió sobre la base de las conversaciones con el profesor, Doctor Richard Antonio Orozco Contreras.

Estamos de acuerdo con la postura del profesor Orozco Contreras cuando afirma que la concepción semántica de las teorías tiene supuestos metafísicos, lo cual dicho sea de paso es aceptado por el estructuralismo científico, así (Moulines, 1996, pp. 3-4); sobre esta cuestión hemos de indicar que dichos presupuestos no dan lugar al abandono de la concepción semántica, como de hecho ocurre en el caso de los dogmas que tratamos esta oportunidad que dan lugar al abandono del empirismo lógico. 
sólo en Perú haya más de 30 millones de individuos que son ejemplificaciones de "hombre". Este es un argumento formal, y no creemos que sea decisivo, pero muestra claramente que puede haber una gran ejemplificación de un concepto y no por ello el mismo se trivializa.

Como es conocido, en "Dos dogmas del empirismo", Quine (1961) demostró que es insostenible las afirmaciones empiristas de la distinción analítico/ sintético y la reducción lógica del significado a la experiencia. Pues bien, si al empleo de la noción de dogma que hace Quine no se le puede entender en el sentido de "verdades divinas". Sí es el caso que los concibe como afirmaciones sin sustento o con sustento aparente. Este tipo de ataques contra el empirismo también lo hizo Hilary Putnam (1962) cuando criticó la distinción términos teóricos/términos observacionales y el concepto de interpretación parcial que eran sostenidos por el empirismo lógico. Ahora, si esto no ha llegado a ser considerado como otros dogmas del empirismo, no es porque Putnam no haya hecho uso de la noción de dogma (de hecho en su trabajo publicado en 1962 no dice nada al respecto), sino que este intento de Putnam fue superado por Frederick Suppe en (1989).

O sea que, aún en el fallido trabajo de Putnam (si aceptamos la respuesta de Suppe), la noción de dogma del empirismo es el de afirmaciones sin sustento o con sustento aparente hechas en el seno del empirismo lógico. Esta noción de dogma del empirismo (como afirmación sin sustento o con sustento aparente hecho en el seno del empirismo lógico), no limita su número de ejemplificaciones y por ende no se trivializa por un número elevado las mismas. Sería el caso de una trivialización (por ambigüedad), si fuera que llamáramos dogmas del empirismo a otras cosas que no sean afirmaciones sin sustento o con sustento aparente.

\section{Conclusiones.}

El cambio más importante a nivel epistemológico del siglo pasado, es el surgimiento de la concepción semántica. La cual a su vez representa, y no podía ser de otro modo, la respuesta a las diversas críticas hechas al empirismo lógico, y que se han denominado como "dogmas". En este caso, existe una clara relación entre los acusados séptimo y octavo dogmas del empirismo y la concepción semántica. Asimismo, ni siquiera las versiones más "liberales" del empirismo lógico podrían haber respondido adecuadamente la crítica de no prestar atención a los aspectos pragmáticos de las teorías (incluyendo la inconmensurabilidad). Lo cual ha sido y es materia de una rigurosa elaboración en el seno de la concepción semántica (e.g. el estructuralismo teórico), evitando con ello las ambigüedades en las que incurriera Thomas S. Kuhn en su momento.

\section{REFERENCIAS}

Awodey, S., E Reck, E. H. (2002). «Completeness and categoricity, Part I: Nineteenth-century Axiomatics to Twentieth-century Metalogic». History and Philosophy of Logic(23), 1-30.

Awodey, S., E Reck, E. H. (2002). «Completeness and categoricity, Part II. Twentieth-Century Metalogic to Twenty-first-Century Semantics》. History and Philosophy of Logic(23), 77-94.

Balzer, W., Moulines, C. U., \& Sneed, J. D. (1987). An architectonic for science. The Structuralist program. Dordrecht: D. Reidel Publishing Company. 
Bernays, P. (1967). «Scope and Limits of Axiomatics». In M. Bunge (Ed.), Studies in the Foundations Methodology and Philosophy of Science (Vol. I. Delaware Seminar in the Foundations of Physics, pp. 188-91). New York: Springer-Verlag.

Carnap, R. (1956). «The methodological character of theoretical concepts». In H. Feigl, \& M. Scriven (Eds.), The Foundations of Science and the Concepts of Psychology and Psychoanalysis (pp. 38-76). Minneapolis: University of Minnesota.

Carnap, R. ( 19558$)$. «Beobachtungssprache und theoretische sprache». Dialectica(12), 236-48.

Carnap, R. (1992 (1963)). Autobiografía intelectual. (C. Castells, Trad.) Barcelona: Paidós Ibérica.

Carnap, R. (2005 (1928)). The Logical Structure of the World and Pseudoproblems in Phylosophy. (R. A. George, Trans.) Chicago: Carus Publishing Company.

Cassini, A. (2006). El juego de los principios. Una introducción al método axiomático. Buenos Aires: AZ.

Chakravartty, A. (2001). «The semantic or model-theoretic view of theories and scientific realism». Synthese, 127 , 325-345.

Coll Mármol, J. A. (2007, Agosto). «McDowell's dogmatic empiricism. Crítica». Revista Hispanoamericana de Filosofía, 39(116), 37-50.

Corcoran, J. (1980). «Categoricity». History and Philosophy of Logic(1), 187 - 207.

Da Costa, N. A., E French, S. (2003). Science and Partial Truth. A Unitary
Approach to Models and Scientific Reasoning. Oxford: Oxford University Press.

Davidson, D. (1973-1974). «On the Very Idea of a Conceptual Scheme». Proceedings and Addresses of the American Philosophical Association, 47, 5-20.

Díez, J. A., E Moulines, C. U. (2008). Fundamentos de Filosofía de la Ciencia (Tercera ed.). Barcelona: Ariel.

Euclid. (1908). The thirteen books of Euclid's elements (Vol. I). (T. L. Heath, Ed., \& T. L. Heath, Trans.) Cambridge: Cambridge University Press.

Feigl, H. (1970). «The 'Orthodox' view of theories: Remarks in defense as well as critique». In M. Radner, $\&$ S. Winokur (Eds.), Minnesota Studies in Philosophy of Science (Vol. 4. 4. Theories $\varepsilon$ Methods of Physics and Psychology, pp. 3-16). Minneapolis: University of Minnesota Press.

Ferreirós, J. (2014).«Introducción». En R. Dedekind, ¿Qué son y para qué sirven los números? (págs. 13-102). Madrid: Universidad Autónoma de Madrid - Alianza Editorial.

Freudenthal, H. (1962).«The main trends in the foundations of geometry in the 19th century». In E. Nagel, P. Suppes, E A. Tarski (Eds.), Logic, Methodology and Philosophy of Science. Proceedings of the 1960 International Congress (pp. 613-621). Standford: Standford University Press.

Giere, R. N. (1988). Explaning Science. A cognitive approach. Chicago and London: The University of Chicago.

Hands, D. W. (2001). Reflection without Rules. Cambridge: Cambridge University Press. 
Hempel, C. G. (1970). «On the "Standard Conception" of Scientific Theories». In M. Radner, \& S. Winokur (Eds.), Minnesota Studies in the Philosophy of Science (Vol. 4. Theories \& Methods of Physics and Psychology, pp. 142-63). Minneapolis: University of Minnesota Press.

Hilbert, D. (1996 [1918]). «Axiomatic thought». In W. B. Ewald (Ed.), From Kant to Hilbert: A Source Book in the Foundations of Mathematics (Vol. II, pp. 1105-15). Oxford: Oxford University Press.

Hilbert, D. (1996 [1922]). «The new grounding of mathematics». In W. Ewald (Ed.), From Kant to Hilbert: A Source Book in the Foundations of Mathematics (Vol. II, pp. 1115-34). Oxford: Oxford University Press.

Hilbert, D. (1996 [1930]). «The Knowledge of Nature». In W. Ewald (Ed.), From Kant to Hilbert. A Source Book in the Foundations of Mathematics (Vol. II, pp. 1157-65). Oxford: Oxford University Press.

Hughes, R. I. (2010). The theoretical practices of physics. Philosophical essays. Oxford et al.: Oxford University Press.

Huntington, E. V. (1902, April). «A Complete Set of Postulates for the Theory of Absolute Continuous Magnitude». Transactions of the American Mathematical Society, 3(2), 264-279.

Ibarra, A. (2012). «La interpretación estructuralista del Statement View». En L. M. Peris-Viñé (Ed.), Filosofía de la Ciencia en Iberoaméica: Matateoría estructural (págs. 82-102). Madrid: Tecnos.

Ibarra, A. (2012). «Réplica al 'Comentario de Pablo Lorenzano'».
En Filosofía de la Ciencia en Iberoamérica: Metateoría estructural (págs. 114-9). Madrid: Tecnos.

Kraft, V. (1966 [1950]). El círculo de Viena. (F. Gracia, Trad.) Madrid: Taurus.

Kripke, S. (2014). «Yet another dogma of empiricism». Philosophy and Phenomenological Research, 1-4. doi:10.1111/phpr.12084

Kuhn, T. S. (1996). The structure of scientific revolutions (Third ed.). Chicago and London: The University of Chicago Press.

Kuhn, T. S. (2000). The Road since Structure. Philosophical Essays, 1970 1993, with an Autobiographical Interview. Chicago and London: The University of Chicago Press.

Lorenzano, P. (2012). «Comentario a "La interpretación estructuralista del Statement View" de Andoni Ibarra». En L. M. Peris-Viñé (Ed.), Filosofía de la Ciencia en Iberoamérica: Metateoría estructural (págs. 102-14). Madrid: Tecnos.

Lorenzano, P. (2013, December). «The semantic conception and the structuralist view of theories: A critique of Suppe's criticisms». Studies in History and Philosophy of Science, 44(4), 600-607.

Masterman, M. (1970). «The nature of a Paradigm». In Criticism and the Growth of Knowledge. Proceedings of the International Colloquium in the Philosophy of Science, London, 1965, volume 4 (pp. 59-89). Cambridge: Cambridge University Press.

Morgan, M. S. (2012). The World in the Model. How Economists Work and Think. Cambridge et al.: Cambridge University Press. 
Mormann, T. (2010). «Enlightenment and Formal Romanticism - Carnap's account of philosophy as explication». In J. Manninen, E F. Stadler (Eds.), The Vienna Circle in the Nordic Countries (pp. 263-79). Springer et al.: Springer.

Morrison, M. (2015). Reconstructing Reality: Models, Mathematics, and Simulations. Oxford: Oxford University Press.

Moulines, C. U. (1982). Exploraciones metacientíficas. Estructura, desarrollo y contenido de la ciencia. Madrid: Alianza.

Moulines, C. U. (1996). «Structuralism: The basic ideas». In W. Balzer, \& C. U. Moulines (Eds.), Structuralist Theory of Science. Focal issues, New results (pp. 1-13). de Gruyter.

Moulines, C. U. (2011). «Cuatro tipos de desarrollo teórico en las ciencias empíricas). Metatheoria, 1(2), 11-27.

Moulines, C. U., E Díez, J. A. (1994). «Theories as Nets: The Caseof Combinatorial Measurement Theory». In P. Humphreys (Ed.), Patrick Suppes: Scientific Philosopher (Vols. 2. Philosophy of Physics, Theory Structure, and Measurement Theory, pp. 275-97). Dordrecht: Kluwer AcademicPublishers.

Putnam, H. (1962). «What theories are not». In E. Nagel, P. Suppes, E A. Tarski (Eds.), Logic, Methodology, and Philosophy of Science. Proceedings of the 1960 International Congress (pp. 240-251). Stanford: Stanford University Press.

Putnam, H. (2002). The collapse of the fact-value dichotomy and other essays. Cambridge and London: Harvard University Press.
Quine, W. v. (1961). «Two Dogmas of empiricism». In W. v. Quine, from a logical point of view (Second ed., pp. 20-46). New York, Hagerstown, San Francisco, London: Harper $\&$ Row, Publishers, Inc.

Reichenbach, H. (1938). Experience and Prediction. An Analysis of the Foundations and the Structure of Knowledge. Chicago: The University of Chicago Press.

Reid, C. (1970). Hilbert. Berlin Heidelberg: Springer-Verlag.

Rodin, A. (2014). Axiomatic Method and Category Theory. Cham: Springer International Publishing.

Salmon, W. (1977). «A Third Dogma of Empiricism». In Basic problems in methodology and linguistics. Part three of the Fifth International Congress of Logic, Methodology, and Philosophy of Science, London, Ontario, Canada - 1975 (pp. 149-166). Dordrecht: Springer.

Schurz, G. (2014). Philosophy of Science: A Unified Approach. New York: Routledge.

Stegmüller, W. (1976 (1973)). The Structure and Dynamics of Theories. (W. Wohlhueter, Trans.) New York: Springer.

Stegmüller, W. (1979). The Structuralist View of Theories. A possible Analogue of the Bourbaki Programme in Physical Science. Berlin, Heidelberg and New York: Springer-Verlag.

Stegmüller, W. (1983). «Erklärung Begründung Kausalität». In W. Stegmüller, Probleme und Resultate der Wissenschaftstheorie und Analytische Philosophie (Vol. Band I. Teil A. Das dritte Dogma des Empirismus. Das ABC der modern Logik und Semantik. Der Begriff der Erklärung und seine Spielarten, pp. 1-190). Berlin, Heidelberg und New York: Springer-Verlag. 
Stegmüller, W. (1983). «Vom dritten bis sechsten (siebsen?) Dogma des Empirismus». Erkenntnis und Wissenschaftstheorie: Akten des 7. Internationalen Wittgenstein-Symposiums, 22-29. Aug. 1982, 232-44.

Stegmüller, W. (1984 (1978)). «Planteamiento combinado de las teorías. Cómo mejorar las interpretaciones históricas del Cambio de Teorías aplicando estructuras de la Teoría de Conjuntos». En G. Radnitzky, $\mathcal{E}$ G. Andersson (Edits.), Estructura y desarrollo de la ciencia (D. Ribes, Trad., págs. 233-64). Alianza Editorial.

Suppe, F. (1977). «The search for Philosophic Understading of Scientific Theories». In F. Suppe (Ed.), The Structure of Scientific Theories (Second ed., pp. 3-241). Urbana: University of Illinois Press.

Suppe, F. (1989). The semantic conception of theories and scientific realism. Urbana: University of Illinois Press.

Suppes, P. (1960). A Comparison of the Meaning and Uses of Models in Mathematics and the Empirical Sciences. Standford: Office of Naval Research.

Suppes, P. (1967). «What is a scientific theory?» In S. Morgenbesser (Ed.), Philosophy of science today (pp. 55-67). New York: Basic Books, Inc.
Suppes, P. (1993 (1979)). «The role of formal methods in the philosophy of science». In P. Suppes, Models and Methods in the Philosophy of Science. Selected Essays (pp. 3-14). Dordrecht: Springer.

Suppes, P. (2002). Representation and Invariance of Scientific Structures (Second ed.). Stanford: Center for the Study of Language and Information.

Tarski, A. (1971 (1953)). «A general method in proofs of undecidability». In A. Tarski, A. Motowski, \& R. M. Robinson, Undecidable Theories (pp. 1-35). Amsterdam: North-Holland Publishing Company.

Torretti, R. (1993). «El método axiomático». En C. U. Moulines (Ed.), La ciencia: estructura y desarrollo (págs. 89-110). Madrid: Trotta.

Van Fraassen, B. C. (1980). The Scientific Image. Oxford: Oxford University Press.

Veblen, O. (1904, March 4). «A system of axioms for Geometry». Transaction of american mathematical society, 5(3), 343-84.

Veblen, O. (1905). «Definition in terms of order alone in the linear continuum and in well-ordered sets». Transactions of the American Mathematical Society, 6(2), 165-71. 
